\title{
On the Effect of Switching, Predation and Harvesting on Systems Consisting of One Predator and Two Prey Species Which Live in Different Habitats
}

\author{
B.S. Bhatt (Corresponding author) \\ Department of Mathematics and Statistics, Faculty of Science and Agriculture \\ The University of the West Indies, St. Augustine, Republic of Trinidad and Tobago \\ E-mail: bal.bhatt@sta.uwi.ed \\ D.R. Owen \\ Department of Mathematics and Statistics, Faculty of Science and Agriculture \\ The University of the West Indies, St. Augustine, Republic of Trinidad and Tobago \\ E-mail: drowentt@yahoo.com \\ R.P. Jaju \\ Department of Computer Science, Faculty of Science \\ The University of Swaziland, P/B 4, Kwaluseni, Kingdom Of Swaziland \\ E-mail: jajurp@uniswa.sz
}

Received: February 23, 2011 Accepted: March 15, 2011 doi:10.5539/jmr.v3n3p12

\begin{abstract}
The present work deals with two prey species living in two habitats and a predator specie which attacks the most abundant prey specie. The harvesting of both prey species is taken into account in the analysis. Non-zero equilibrium states have been examined with regard to their stability and the conditions for stability have been obtained. Using as a bifurcation parameter the conversion rate of prey to predator, conditions for a bifurcation to occur are obtained. A Hopf bifurcation theorem has been derived. In the investigations we used six forms of predation and harvesting rates to analyze the theory, however, we display graphical results for only one particular case.
\end{abstract}

Keywords: Prey, Predator, Predation/Harvesting, Switching, Stability, Hopf Bifurcation

\section{Introduction}

Good functional representation is necessary in the analytical treatment of predator-prey systems. If harvesting of the preys is to be included then we must also have a representation of that interaction.

In constructing interaction functions it is useful in many cases to get a general form for the function together with any general conditions one may wish them to satisfy, then using elementary functions, construct specific functions. This will be our guide for the determination of the interaction functions. Our present model of predator-prey systems will be based on the models of (Bhatt, 2000, p 3133-3137), (Bhatt, 2002, p 2304-2309) and (Owen, 2010, p 281-295).

In (Bhatt, 2000, p 3133-3137), systems of two preys which live in two different habitats and one predator specie which may switch towards the most abundant prey specie is treated. In (Bhatt, 2002, p 2304-2309), the authors extend (Bhatt, 2000 , p 3133-3137) by including general predator-prey interactions which allow the predator to interact in the same way with the two preys. That is, the predatory rates are given the same functional form. In (Owen, 2010, p 281-295) predator interference is allowed.

Our objective in the present work is to extend the above works by allowing harvesting of the preys. This is carried out by considering general harvesting functions in addition to the general predatory functions. We determine the equilibrium states, their stability conditions and Hopf bifurcation points and we present a Hopf bifurcation theorem.

In our applications we consider hypothetical systems and examine only those equilibrium states where all species coexist.

In our investigations we examined the following hypothetical systems where predator and harvesting functions are dependent only on the ratio of the prey populations. The systems had only one equilibrium state and we derived conditions for the stability of these states. 
The hypothetical systems have the following predatory and harvesting functions:

System 1: $\quad k_{1}\left(\frac{x_{2}}{x_{1}}\right)=\frac{1}{1+\left(\frac{x_{2}}{x_{1}}\right)^{n}}, h_{1}\left(\frac{x_{2}}{x_{1}}\right)=1, k_{2}\left(\frac{x_{1}}{x_{2}}\right)=\frac{1}{1+\left(\frac{x_{1}}{x_{2}}\right)^{n}}, h_{2}\left(\frac{x_{1}}{x_{2}}\right)=1$,

System 2: $\quad k_{1}\left(\frac{x_{2}}{x_{1}}\right)=\frac{1}{\exp \left(\frac{x_{2}}{x_{1}}\right)^{n}}, h_{1}\left(\frac{x_{2}}{x_{1}}\right)=1, k_{2}\left(\frac{x_{1}}{x_{2}}\right)=\frac{1}{\exp \left(\frac{x_{1}}{x_{2}}\right)^{n}}, h_{2}\left(\frac{x_{1}}{x_{2}}\right)=1$,

System 3: $\quad k_{1}\left(\frac{x_{2}}{x_{1}}\right)=h_{1}\left(\frac{x_{2}}{x_{1}}\right)=\frac{1}{1+\left(\frac{x_{2}}{x_{1}}\right)^{n}}, k_{2}\left(\frac{x_{1}}{x_{2}}\right)=h_{2}\left(\frac{x_{1}}{x_{2}}\right)=\frac{1}{1+\left(\frac{x_{1}}{x_{2}}\right)^{n}}$,

System 4: $\quad k_{1}\left(\frac{x_{2}}{x_{1}}\right)=h_{1}\left(\frac{x_{2}}{x_{1}}\right)=\frac{1}{\exp \left(\frac{x_{2}}{x_{1}}\right)^{n}}, k_{2}\left(\frac{x_{1}}{x_{2}}\right)=h_{2}\left(\frac{x_{1}}{x_{2}}\right)=\frac{1}{\exp \left(\frac{x_{1}}{x_{2}}\right)^{n}}$,

System 5: $\quad k_{1}\left(\frac{x_{2}}{x_{1}}\right)=\frac{1}{1+\left(\frac{x_{2}}{x_{1}}\right)^{n}}, h_{1}\left(\frac{x_{2}}{x_{1}}\right)=\frac{1}{\exp \left(\frac{x_{2}}{x_{1}}\right)^{n}}, k_{2}\left(\frac{x_{1}}{x_{2}}\right)=\frac{1}{1+\left(\frac{x_{1}}{x_{2}}\right)^{n}}, h_{2}\left(\frac{x_{1}}{x_{2}}\right)=\frac{1}{\exp \left(\frac{x_{1}}{x_{2}}\right)^{n}}$,

System 6: $\quad k_{1}\left(\frac{x_{2}}{x_{1}}\right)=\frac{1}{\exp \left(\frac{x_{2}}{x_{1}}\right)^{n}}, h_{1}\left(\frac{x_{2}}{x_{1}}\right)=\frac{1}{1+\left(\frac{x_{2}}{x_{1}}\right)^{n}}, k_{2}\left(\frac{x_{1}}{x_{2}}\right)=\frac{1}{\exp \left(\frac{x_{1}}{x_{2}}\right)^{n}}, h_{2}\left(\frac{x_{1}}{x_{2}}\right)=\frac{1}{1+\left(\frac{x_{1}}{x_{2}}\right)^{n}}$,

where $n$ is a positive integer and the functions $k_{i}, h_{i}, i=1,2$ represent predatory and harvesting rates, respectively, which occur in the equations defining the model.

We point out that the parameters used in our numerical calculations do not represent any real situation and are used for illustrative purposes only. These values were obtained by careful guessing. Of the six systems examined we graphically display the results of only System 5.

In Section 2 we give the equations defining our model. Section 3 is devoted to the stability of equilibrium states, while Section 4 contains the Hopf bifurcation analysis. In Section 5 we consider one application while Section 6 contains the results.

\section{System of equations defining the Model}

The predator-prey model in which the predator can switch towards the most abundant prey specie and which allows general predatory and harvesting rates is defined by the equations:

$$
\begin{aligned}
\frac{d x_{1}}{d t} & =\left(\alpha_{1}-\epsilon_{1}\right) x_{1}+\epsilon_{2} p_{21} x_{2}-\beta_{1} x_{1} y k_{1}\left(\frac{x_{2}}{x_{1}}\right)-x_{1} \delta_{1} h_{1}\left(\frac{x_{2}}{x_{1}}\right) \\
\frac{d x_{2}}{d t} & =\left(\alpha_{2}-\epsilon_{2}\right) x_{2}+\epsilon_{1} p_{12} x_{1}-\beta_{2} x_{2} y k_{2}\left(\frac{x_{1}}{x_{2}}\right)-x_{2} \delta_{2} h_{2}\left(\frac{x_{1}}{x_{2}}\right) \\
\frac{d y}{d t} & =\left[-\mu+c_{1} \beta_{1} x_{1} k_{1}\left(\frac{x_{2}}{x_{1}}\right)+c_{2} \beta_{2} x_{2} k_{2}\left(\frac{x_{1}}{x_{2}}\right)\right] y
\end{aligned}
$$

where

$x_{i}$ : represents the prey population in the two different habitats,

$\delta_{i}$ : represents the harvesting rate of the prey population in the two different habitats,

$y$ : represents the abundance of predator species,

$\beta_{i}$ : the predator response rates towards the prey $x_{i}$,

$c_{i}$ : the rate of conversion of prey to predator,

$\epsilon_{i}$ : inversion barrier strength in going out of the habitat,

$p_{i j}$ : the probability of successful transition from the $i^{\text {th }}$ habitat to the $j^{\text {th }}$ habitat,

$\alpha_{i}$ : $\quad$ specific growth rate of the prey in the absence of predation,

$\mu: \quad$ per capita death rate of the predator and 
also

$k_{1}\left(\frac{x_{2}}{x_{1}}\right), h_{1}\left(\frac{x_{2}}{x_{1}}\right)$ and $k_{2}\left(\frac{x_{1}}{x_{2}}\right), h_{2}\left(\frac{x_{1}}{x_{2}}\right)$ are general positive functions of the ratios $\frac{x_{2}}{x_{1}}$ and $\frac{x_{1}}{x_{2}}$ respectively. These functions are assued to satisfy certain general conditions which are given by Assumptions 1 - 3 in section 3 . All the coefficients, $\delta_{i}, \beta_{i}, c_{i}, \epsilon_{i}, p_{i j}, \alpha_{i}$, and $\mu$ are positive.

\section{Stability of the equilibrium}

Let $\left(X_{1}, X_{2}, Y\right)$ be the equilibrium states of the system described by equations (1), when all species coexist. On solving the equilibrium equations, we get the following expressions for $\left(X_{1}, X_{2}, Y\right)$ :

$$
\begin{aligned}
X_{1} & =\frac{\mu \bar{X}}{c_{1} \beta_{1} \bar{X} k_{1}\left(\frac{1}{\bar{X}}\right)+c_{2} \beta_{2} k_{2}(\bar{X})}, \\
X_{2} & =\frac{\mu}{c_{1} \beta_{1} \bar{X} k_{1}\left(\frac{1}{\bar{X}}\right)+c_{2} \beta_{2} k_{2}(\bar{X})}, \\
Y & =\frac{\left(\alpha_{1}-\epsilon_{1}\right) \bar{X}+\epsilon_{2} p_{21}-\delta_{1} \bar{X} h_{1}\left(\frac{1}{\bar{X}}\right)}{\beta_{1} \bar{X} k_{1}\left(\frac{1}{\bar{X}}\right)}, \\
Y & =\frac{\left(\alpha_{2}-\epsilon_{2}\right)+\epsilon_{1} p_{12} \bar{X}-\delta_{2} h_{2}(\bar{X})}{\beta_{2} k_{2}(\bar{X})}
\end{aligned}
$$

We note that there are two expressions for $Y$, hence as it stands, the equilibrium point $E=\left(X_{1}, X_{2}, Y\right)$ may or may not exist and even if it exists it may not represent real populations. The following Lemma provides conditions for the equilibrium point, $\left(X_{1}, X_{2}, Y\right)$ to exist and to represent real populations.

Lemma 1: The equilibrium point, $\left(X_{1}, X_{2}, Y\right)$ of equations (1), exists and represents real populations if $\bar{X}\left(=\frac{X_{1}}{X_{2}}\right)$ satisfies $\left[\left\{\left(\alpha_{1}-\epsilon_{1}-\delta_{1} h_{1}\left(\frac{1}{\bar{X}}\right)\right\} \bar{X}+\epsilon_{2} p_{21}\right] \beta_{2} k_{2}(\bar{X})=\right.$

$$
\beta_{1} \bar{X} k_{1}\left(\frac{1}{\bar{X}}\right)\left(\alpha_{2}-\epsilon_{2}+\epsilon_{1} p_{12} \bar{X}-\delta_{2} h_{2}(\bar{X})\right)
$$

and

$$
\frac{\epsilon_{2}-\alpha_{2}+\delta_{2} h_{2}(\bar{X})}{\epsilon_{1} p_{12}}<\bar{X}<\frac{\epsilon_{2} p_{21}}{\epsilon_{1}-\alpha_{1}+\delta_{1} h_{1}\left(\frac{1}{\bar{X}}\right)}
$$

Proof: Equating the two expressions for $Y$, thus producing equation (3), ensures existence of the equilibrium point while inequalities (4) make $Y>0$ so as to represent a real population.

\subsection{Assumptions and general stability conditions}

The stability of the equilibrium point, $\left(X_{1}, X_{2}, Y\right)$ where $X_{1}, X_{2}, Y$ are all positive will now be considered. We begin by making the following three assumptions:

[Assumption 1:]

Each predatory / harvesting function $k_{i}\left(\frac{x_{i}}{x_{j}}\right), h_{i}\left(\frac{x_{i}}{x_{j}}\right) i=1,2$, is positive, smooth and has a Taylor expansion in the two variables $x_{1}, x_{2}$ about the point $\left(X_{1}, X_{2}\right)$ where $\left(X_{1}, X_{2}, Y\right)$ is an equilibrium point of the system of equations (1).

[Assumption 2:]

The predatory / harvesting functions, $k_{i}, h_{i} i=1,2$ satisfy the following inequalities

$$
k_{1}^{\prime}\left(\frac{x_{2}}{x_{1}}\right)<0, h_{1}^{\prime}\left(\frac{x_{2}}{x_{1}}\right)<0, k_{2}^{\prime}\left(\frac{x_{1}}{x_{2}}\right)<0, h_{2}^{\prime}\left(\frac{x_{1}}{x_{2}}\right)<0
$$


where $x_{1}, x_{2}$ are positive and $k_{1}^{\prime}(z), z=\frac{x_{i}}{x_{j}}$, denotes the derivative of $k_{1}$ with respect to $z$, etc.

[Assumption 3: (The Switching Assumption)]

The predator rate functions, for $x_{1}, x_{2}$ both positive, satisfy:

1. for $\frac{x_{1}}{x_{2}}>>1$, i.e. as $\frac{x_{1}}{x_{2}} \rightarrow \infty, k_{1}\left(\frac{x_{2}}{x_{1}}\right) \rightarrow 1, k_{2}\left(\frac{x_{1}}{x_{2}}\right) \rightarrow 0$

and

2. for $\frac{x_{2}}{x_{1}}>>1$, i.e. as $\frac{x_{2}}{x_{1}} \rightarrow \infty, k_{1}\left(\frac{x_{2}}{x_{1}}\right) \rightarrow 0, k_{2}\left(\frac{x_{1}}{x_{2}}\right) \rightarrow 1$.

Assumption 3, the switching assumption, reflects the type of feeding mechanism which is adapted by the predators. This is a switching mechanism in our present situation.

\subsection{Stability analysis}

To consider the stability of the equilibrium point, $\left(X_{1}, X_{2}, Y\right)$, we linearize the equations (1) by considering a small perturbation about the equilibrium point i.e. by substituting $x_{1}=X_{1}+u, x_{2}=X_{2}+v, y=Y+w$, in all functions in equations (1) and expanding them using Taylorś expansion and neglecting second and higher order terms in $u, v, w$.

Defining $w_{1}, w_{2}, A, B, C$ and $D$ as follows

$$
\begin{aligned}
w_{1} & =\beta_{1} X_{1} k_{1}\left(\frac{X_{2}}{X_{1}}\right), w_{2}=\beta_{2} X_{2} k_{2}\left(\frac{X_{1}}{X_{2}}\right) \\
A & =c_{1} \beta_{1} k_{1}\left(\frac{X_{2}}{X_{1}}\right)+c_{1} \beta_{1}\left\{-\frac{X_{2}}{X_{1}} k_{1}^{\prime}\left(\frac{X_{2}}{X_{1}}\right)\right\}+c_{2} \beta_{2} k_{2}^{\prime}\left(\frac{X_{1}}{X_{2}}\right), \\
B & =c_{1} \beta_{1} k_{1}^{\prime}\left(\frac{X_{2}}{X_{1}}\right)+c_{2} \beta_{2}\left\{-\frac{X_{1}}{X_{2}} k_{2}^{\prime}\left(\frac{X_{1}}{X_{2}}\right)\right\}+c_{2} \beta_{2} k_{2}\left(\frac{X_{1}}{X_{2}}\right), \\
C & =\epsilon_{1} p_{12}-\beta_{2} Y k_{2}^{\prime}\left(\frac{X_{1}}{X_{2}}\right)-\delta_{2} h_{2}^{\prime}\left(\frac{X_{1}}{X_{2}}\right) \\
D & =\epsilon_{2} p_{21}-\beta_{1} Y k_{1}^{\prime}\left(\frac{X_{2}}{X_{1}}\right)-\delta_{1} h_{1}^{\prime}\left(\frac{X_{2}}{X_{1}}\right)
\end{aligned}
$$

we see that the linearized form of equations (1) can be written as

$$
\frac{d V}{d t}=J V
$$

where, $V=\left(\begin{array}{c}u \\ v \\ w\end{array}\right)$. The corresponding characteristic equation is given by

$$
|J|=\left|\begin{array}{ccc}
-\frac{D}{\bar{X}}-\lambda & D & -w_{1} \\
C & -C \bar{X}-\lambda & -w_{2} \\
A Y & B Y & -\lambda
\end{array}\right|=0 .
$$

where $A, B, C, D$ are given by equations (6).

We may write equation (7) as

$$
\lambda^{3}+b_{1} \lambda^{2}+b_{2} \lambda+b_{3}=0
$$

where

$$
\begin{aligned}
& b_{1}=\bar{X} C+\frac{D}{\bar{X}} \\
& b_{2}=Y\left(A w_{1}+B w_{2}\right), \\
& b_{3}=\frac{Y}{\bar{X}}(A \bar{X}+B)\left(D w_{2}+C \bar{X} w_{1}\right) .
\end{aligned}
$$


For stability of the equilibrium to be ensured the eigenvalue solutions, $\lambda$, must have negative real parts. The RouthHurwitz criteria provide conditions for this to happen. These criteria tell us that the eigenvalues will have negative real parts if and only if

$$
\begin{gathered}
b_{1}>0, \\
b_{3}>0, \\
b_{1} b_{2}-b_{3}>0 .
\end{gathered}
$$

From Assumption 2 we see that $C>0, D>0$, hence $b_{1}>0$. From equations (9) we see also that $b_{3}$ is positive if $\bar{X} A+B>0$ since $\bar{X}, Y, w_{1}, w_{2}, C, D$ are all positive. Now with the help of equations (6) we can indeed show that

$$
\bar{X} A+B=\frac{\mu}{X_{2}}
$$

which tells us that $b_{3}>0$.

In the light of the above results we can write the following general theorem:

Theorem 1: If the functions $k_{i}, h_{i}$ obey Assumptions 1, 2 and the conditions of the above Lemma 1 hold and A, B, C, D are defined by equations (6) with $k_{i}$,' given as in Assumption (2), then the stability of the equilibrium point $\left(X_{1}, X_{2}, Y\right)$ is assured if and only if

$$
\Delta \equiv b_{1} b_{2}-b_{3}=\left\{\beta_{2} k_{2}\left(\frac{X_{1}}{X_{2}}\right)-\beta_{1} k_{1}\left(\frac{X_{2}}{X_{1}}\right)\right\}\left(B C X_{1}-A D X_{2}\right)>0 .
$$

\section{Hopf Bifurcation}

Assuming $A>0, B>0$ and using the rate of conversion of prey to predator, $c_{1}$, as the bifurcation parameter, we search for Hopf bifurcations with respect to the equations (1).

Using Assumption 2 with $A>0, B>0$, we see, that $C, D, b_{1}, b_{2}, b_{3}$ are all positive. (Actually, $b_{3}$ was shown to be positive without assuming $A>0, B>0$ in the previous section 3).

Also, it is seen that $\bar{X}, Y, C, D$ are all independent of the parameters $c_{1}$ and $c_{2}$. That $C, D$ are independent of $c_{1}, c_{2}$ can be seen from equations (6) and by noting that -

$k_{2}^{\prime}\left(\frac{X_{1}}{X_{2}}\right), h_{2}^{\prime}\left(\frac{X_{1}}{X_{2}}\right), k_{1}^{\prime}\left(\frac{X_{2}}{X_{1}}\right), h_{1}^{\prime}\left(\frac{X_{2}}{X_{1}}\right)$ are functions of $\bar{X}$ which is independent of $c_{1}, c_{2}$. We also see that $b_{1}, w_{1}, w_{2}$ are independent of $c_{1}, c_{2}$.

In the rest of this section we shall follow the Hopf bifurcation analysis as in (Bhatt, 2002, p 2304-2309) . We shall not, however, reproduce the initial part of the analysis.

For $c_{1} \in\left(\bar{c}_{1}-\epsilon, \bar{c}_{1}+\epsilon\right), \epsilon$ small, and $\bar{c}_{1}$ a positive root of $b_{1} b_{2}=b_{3}$, we can write the general roots of eigenvalue equation (8) as

$$
\lambda_{1}\left(c_{1}\right)=u\left(c_{1}\right)+i v\left(c_{1}\right), \lambda_{2}\left(c_{1}\right)=u\left(c_{1}\right)-i v\left(c_{1}\right), \lambda_{3}\left(c_{1}\right)=-b_{1}\left(c_{1}\right) .
$$

Now from (Massden, 1975), we must verify the transversality condition

$$
\left.\frac{d u}{d c_{1}}\right|_{c_{1}=\bar{c}_{1}} \neq 0
$$

We can show, as in (Bhatt, 2002, p 2304-2309), that

$$
\frac{d u}{d c_{1}}=-\frac{2 b_{2}\left(b_{1} b_{2}^{\prime}-b_{3}^{\prime}\right)}{4\left(b_{2}^{2}+b_{1}^{2} b_{2}\right)}
$$

where $b_{2}^{\prime}=\frac{d b_{2}}{d c_{1}}, b_{3}^{\prime}=\frac{d b_{3}}{d c_{1}}$. We shall use this notation for the derivative w.r.t. $c_{1}$ in what follows.

To show Hopf bifurcation at $c_{1}=\bar{c}_{1}$ we must show that at $c_{1}=\bar{c}_{1}$

$$
b_{1} b_{2}^{\prime}-b_{3}^{\prime} \neq 0
$$

because the denominator of $\frac{d u}{d c_{1}}$ is positive and $b_{2}$ is also positive. 
Since $\bar{X}, Y, C, D$ are independent of $c_{1}, c_{2}$ we can show that

$$
b_{1} b_{2}^{\prime}-b_{3}^{\prime}=\frac{Y}{\bar{X}}\left(w_{2} \bar{X}-w_{1}\right)\left(B^{\prime} C \bar{X}-A^{\prime} D\right) .
$$

Now from equations (6) and Assumption 2 we see that $A^{\prime}>0, B^{\prime}<0, C>0$ hence $B^{\prime} C \bar{X}-A^{\prime} D<0$. Therefore

$$
\left.\frac{d u}{d c_{1}}\right|_{c_{1}=\bar{c}_{1}} \neq 0 \text {. }
$$

provided $\bar{X} \neq \frac{w_{1}}{w_{2}}$.

We can write these results in the following theorem:

Theorem 2: Let the predatory / harvesting functions, $k_{i}, h_{i}, i=1,2$, satisfy Assumptions 1 and 2 above, and let the conditions of the Lemma 1 hold. Let also A, B defined by equations (6), be both positive. If $\bar{c}_{1}$ is a positive root of the equation $b_{1} b_{2}=b_{3}$, where $b_{i}, i=1,2,3$ are given by equation (9), then we have a Hopf bifurcation as $c_{1}$ passes through $\bar{c}_{1}$ provided $\beta_{2} k_{2}\left(\frac{X_{1}}{X_{2}}\right) \neq \beta_{1} k_{1}\left(\frac{X_{2}}{X_{1}}\right)$.

Performing a similar analysis with $c_{2}$ (the rate of conversion of the prey in the second habitat to the predator) as the variable parameter, we shall get a similar result.

\section{Applications}

We examined six hypothetical systems which are defined by the predatory and harvesting functions given in the Introduction. The results support the present theory. However, we display graphically the results for only one of these. This is the system given by System 5 in the Introduction. In System 5 the functions are interpreted as defining a system where the predators may attack the two types of preys in the same way while harvesting of the preys is conducted in a similar but different way. The equilibrium point $\left(X_{1}, X_{2}, Y\right)$ of this system is such that:

$$
\begin{aligned}
X_{1} & =\frac{\mu \bar{X}}{c_{1} \beta_{1} k\left(\frac{1}{\bar{X}}\right) \bar{X}+c_{2} \beta_{2} k(\bar{X})}, \\
X_{2} & =\frac{\mu}{c_{1} \beta_{1} k\left(\frac{1}{\bar{X}}\right) \bar{X}+c_{2} \beta_{2} k(\bar{X})}, \\
Y & =\frac{\alpha_{2}-\epsilon_{2}+\epsilon_{1} p_{12} \bar{X}-\delta_{2} h(\bar{X})}{k(\bar{X}) \beta_{2}} \\
Y & =\frac{\left(\alpha_{1}-\epsilon_{1}\right)+\frac{\epsilon_{2} p_{21}}{\bar{X}}-\delta_{1} h\left(\frac{1}{\bar{X}}\right)}{k\left(\frac{1}{\bar{X}}\right) \beta_{1}}
\end{aligned}
$$

where

$$
\begin{gathered}
\bar{X}=\frac{X_{1}}{X_{2}}, k\left(\frac{1}{\bar{X}}\right)=\frac{\bar{X}^{n}}{1+\bar{X}^{n}}, k(\bar{X})=\frac{1}{1+\bar{X}^{n}}, \\
h\left(\frac{1}{\bar{X}}\right)=\exp \left(-\frac{1}{\bar{X}^{n}}\right), h(\bar{X})=\exp \left(-\bar{X}^{n}\right)
\end{gathered}
$$

and since the conditions of the Lemma 1 are satisfied, $\bar{X}$ satisfies:

$\bar{X} \beta_{1} k\left(\frac{1}{\bar{X}}\right)\left(\alpha_{2}-\epsilon_{2}+\epsilon_{1} p_{12} \bar{X}-\delta_{2} h(\bar{X})\right)=$

$$
\beta_{2} k(\bar{X})\left[\left\{\alpha_{1}-\epsilon_{1}-\delta_{1} h\left(\frac{1}{\bar{X}}\right)\right\} \bar{X}+\epsilon_{2} p_{21}\right]
$$

and

$$
\frac{\delta_{2} h(\bar{X})+\epsilon_{2}-\alpha_{2}}{\epsilon_{1} p_{12}}<\bar{X}<\frac{\epsilon_{2} p_{21}}{\delta_{1} h\left(\frac{1}{\bar{X}}\right)+\epsilon_{1}-\alpha_{1}} .
$$


From equation (10) stability of the equilibrium point is assured if and only if

$$
\Delta \equiv b_{1} b_{2}-b_{3}=\left\{k\left(\frac{1}{\bar{X}}\right) \beta_{1}-k(\bar{X}) \beta_{2}\right\}(A D-B C \bar{X})>0
$$

where

$$
\begin{aligned}
A & =c_{1} \beta_{1} k\left(\frac{1}{\bar{X}}\right)+c_{1} \beta_{1}\left(\frac{n\left[k\left(\frac{1}{\bar{X}}\right)\right]^{2}}{\bar{X}^{n}}\right)-c_{2} \beta_{2}\left(n \bar{X}^{n-1}[k(\bar{X})]^{2}\right), \\
B & =c_{1} \beta_{1}\left(\frac{-n k\left(\frac{1}{\bar{X}}\right)}{\bar{X}^{n-1}}\right)+c_{2} \beta_{2}\left(n \bar{X}^{n}[k(\bar{X})]^{2}\right)+c_{2} \beta_{2} k(\bar{X}), \\
C & =\epsilon_{1} p_{12}+\beta_{2} Y\left(n \bar{X}^{n-1}[k(\bar{X})]^{2}\right)+\delta_{2}\left(n \bar{X}^{n-1} h(\bar{X})\right), \\
D & =\epsilon_{2} p_{21}+\beta_{1} Y\left(\frac{n\left[k\left(\frac{1}{\bar{X}}\right)\right]^{2}}{\bar{X}^{n-1}}\right)+\delta_{1}\left(\frac{n h\left(\frac{1}{\bar{X}}\right)}{\bar{X}^{n-1}}\right) .
\end{aligned}
$$

In light of Theorem 2 we can write the following corollary for System 5:

Corollary: Let the predatory / harvesting functions $k_{i}, h_{i}, i=1,2$, be defined by System 5 in the Introduction thus satisfying Assumptions 1 and 2. Also let the conditions of the Lemma 1 hold. If A, B defined by equations (15) are both positive and if $\bar{c}_{1}$ is a positive root of the equation $b_{1} b_{2}=b_{3}$, where $b_{i}, i=1,2,3$ are given by equations (9), then we have a Hopf bifurcation as $c_{1}$ passes through $\bar{c}_{1}$ provided $\beta_{1} X_{1}{ }^{n} \neq \beta_{2} X_{2}{ }^{n}$.

\section{Numerical Results - Both Predatory Rates Multiplicative \& Both Harvesting Rates Exponential}

Equations (1) were integrated for the six Systems mentioned in the Introduction using as initial conditions the corresponding equilibrium values. To illustrate the theory, intervals of stability / instability and bifurcation points of non-zero equilibrium states, using equations (11) to equations (15) with $n=1, n=2$ and $n=3$ and $c_{1}$ and $c_{2}$ as the variable parameters are shown in Table-1. The behavior of the populations with time, for only two parameter sets are displayed graphically in Fig.1 and Fig.2. The results of all sets examined, support the theory. We chose the following parameter sets:

1. $\mu=0.01, \alpha_{1}=0.015, \alpha_{2}=0.025, \beta_{1}=0.01, \beta_{2}=0.02, p_{12}=0.3, p_{21}=0.2, \epsilon_{1}=0.02, \epsilon_{2}=0.03, \delta_{1}=0.0001$, $\delta_{2}=0.0005, c_{1}=0.1, c_{2}=0.1, n=1$,

2. $\mu=0.01, \alpha_{1}=0.015, \alpha_{2}=0.025, \beta_{1}=0.02, \beta_{2}=0.01, p_{12}=0.3, p_{21}=0.2, \epsilon_{1}=0.02, \epsilon_{2}=0.03, \delta_{1}=0.0001$, $\delta_{2}=0.0005, c_{1}=0.1, c_{2}=0.4, n=2$.

NOTE: Table-1 appears here.

It may be observed that for all the data values considered in Table-1, the interval of stability decreases when the value of $\mathrm{n}$ increases.

The behavior of the populations with time are graphically displayed in Figure 1 and Figure 2.

It is interesting to observe that in the cases we examined, when there is an instability and the populations of the predators have a 'maximum', i.e. increase, the population of the preys show a 'minimum'. That is, it appears that some of the preys are 'converted' to predators and then afterwards the prey population receive 'reinforcements'.

\section{Bifurcation figures}

If we fix $c_{1}$ and all the other parameters and treat $c_{2}$ as the bifurcation parameter, we can determine the value of $c_{2}$ where bifurcation takes place. Let us denote this value of $c_{2}$ by $\operatorname{Bifpt}\left(c_{2}\right)$.

The effect of different values of $c_{1}$ and $\alpha_{2}\left(\beta_{2}\right)$, while keeping all of the other parameters fixed at $\mu=0.01, \alpha_{1}=$ $0.015, \beta_{1}=0.01, p_{12}=0.3, p_{21}=0.2, \epsilon_{1}=0.02, \epsilon_{2}=0.03, \delta_{1}=0.0001, \delta_{2}=0005, n=1$, on $\operatorname{Bifpt}\left(c_{2}\right)$ was examined and is displayed graphically.

Figure 3 shows the effect on $\operatorname{Bifpt}\left(c_{2}\right)$ of different values of $c_{1}$ and four values of $\alpha_{2}$, namely 0.025, 0.03,0.035 and 0.04. The values of the other parameters being as above together with $\beta_{2}=0.02$.

Figure 4 shows the effect on $\operatorname{Bifpt}\left(c_{2}\right)$ of different values of $c_{1}$ and four values of $\beta_{2}$, namely $0.01,0.015,0.02$, and 0.03 . The values of the other parameters again being as above together with $\alpha_{2}=0.025$. 
In Figure 3 and figure 4, we plotted the bifurcation point $\left(c_{1}, \operatorname{Bifpt}\left(c_{2}\right)\right)$ for different values of $c_{1}, \alpha_{2}\left(\beta_{2}\right)$ and were able to draw straight lines through the different sets of points thus suggesting that for the system with $k_{i}, h_{i}$, defined by System 5 , $\operatorname{Bifpt}\left(c_{2}\right)$, in both cases, varies linearly with $c_{1}$ in the interval considered.

NOTE: Fig 1 from file (p18f1.eps) appears here.

NOTE: Fig 2 from file (p18f4.eps) appears here.

NOTE: Fig 3 from file (alpha.eps) appears here

NOTE: Fig 4 from file (beta.eps) appears here

We feel that our work is of interest in the sense that we have used general predatory and harvesting rate functions, hence it will compliment other existing works.

\section{Acknowledgements}

The authors are extremely grateful to the reviewers for their valuable comments and suggestions.

\section{References}

Bhatt B., Khan Q. , Jaju R. (2000). Switching of predation on a small size prey species living in two different habitats. J. Phys. Soc. Japan, 69(9), 3133 - 3137.

Bhatt B., Owen D. , Jaju R. (2002). Switching effect of predation on prey species living in different habitats with arbitrary predatory rates. J. Phys. Soc. Japan, 71(9), $2304-2309$.

Marsden, J. , McKracken M. (1975). The Hopf Bifurcation and its applications. New York: Springer-Verlag.

Owen D. , Jaju R. , Bhatt B. (2010). Switching of predation on prey species in the presence of predator interference-II. Int. J. of Pure and Appl. Math, 61(3), 281 - 295. 
Table 1. ( $k_{1}, k_{2}$-multiplicative, $h_{1}, h_{2}$-exponential)

\begin{tabular}{|c|c|c|c|c|c|}
\hline$\beta_{1}$ & $\beta_{2}$ & $n$ & STABLE & UNSTABLE & BIF-PT \\
\hline & & & $c_{2}=0.1$ & $\delta_{2}=0.0005$ & \\
\hline \multirow[t]{3}{*}{0.01} & 0.02 & 1 & $0 \leq c_{1}<0.200962$ & $c_{1} \geq 0.200963$ & 0.200962 \\
\hline & & 2 & $0 \leq c_{1}<0.197796$ & $c_{1} \geq 0.197797$ & 0.197796 \\
\hline & & 3 & $0 \leq c_{1}<0.196620$ & $c_{1} \geq 0.196621$ & 0.196620 \\
\hline \multirow[t]{4}{*}{0.02} & 0.01 & 1 & $c_{1}>0.050625$ & $0 \leq c_{1} \leq 0.050624$ & 0.050625 \\
\hline & & 2 & $c_{1}>0.051273$ & $0 \leq c_{1} \leq 0.051272$ & 0.051273 \\
\hline & & 3 & $c_{1}>0.051486$ & $0 \leq c_{1} \leq 0.051485$ & 0.051486 \\
\hline & & & $c_{2}=0.1$ & $\delta_{2}=0.0002$ & \\
\hline \multirow[t]{3}{*}{0.01} & 0.02 & 1 & $0 \leq c_{1}<0.199658$ & $c_{1} \geq 0.199659$ & 0.199658 \\
\hline & & 2 & $0 \leq c_{1}<0.196497$ & $c_{1} \geq 0.196498$ & 0.196497 \\
\hline & & 3 & $0 \leq c_{1}<0.195308$ & $c_{1} \geq 0.195309$ & 0.195308 \\
\hline \multirow[t]{4}{*}{0.02} & 0.01 & 1 & $c_{1}>0.050306$ & $0 \leq c_{1} \leq 0.050305$ & 0.050306 \\
\hline & & 2 & $c_{1}>0.051071$ & $0 \leq c_{1} \leq 0.051070$ & 0.051071 \\
\hline & & 3 & $c_{1}>0.051356$ & $0 \leq c_{1} \leq 0.051355$ & 0.051356 \\
\hline & & & $c_{1}=0.1$ & $\delta_{2}=0.0005$ & \\
\hline \multirow[t]{3}{*}{0.01} & 0.02 & 1 & $c_{2}>0.049762$ & $0 \leq c_{2} \leq 0.049761$ & 0.049762 \\
\hline & & 2 & $c_{2}>0.050558$ & $0 \leq c_{2} \leq 0.050557$ & 0.050558 \\
\hline & & 3 & $c_{2}>0.050860$ & $0 \leq c_{2} \leq 0.050859$ & 0.050860 \\
\hline \multirow[t]{4}{*}{0.02} & 0.01 & 1 & $0 \leq c_{2}<0.197534$ & $c_{2} \geq 0.197535$ & 0.197534 \\
\hline & & 2 & $0 \leq c_{2}<0.195035$ & $c_{2} \geq 0.195036$ & 0.195035 \\
\hline & & 3 & $0 \leq c_{2}<0.194230$ & $c_{2} \geq 0.195231$ & 0.195230 \\
\hline & & & $c_{1}=0.1$ & $\delta_{2}=0.0002$ & \\
\hline \multirow[t]{3}{*}{0.01} & 0.02 & 1 & $c_{2}>0.050086$ & $0 \leq c_{2} \leq 0.050085$ & 0.050086 \\
\hline & & 2 & $c_{2}>0.050892$ & $0 \leq c_{2} \leq 0.050891$ & 0.050892 \\
\hline & & 3 & $c_{2}>0.051202$ & $0 \leq c_{2} \leq 0.051201$ & 0.051202 \\
\hline \multirow[t]{3}{*}{0.02} & 0.01 & 1 & $0 \leq c_{2}<0.198786$ & $c_{2} \geq 0.198787$ & 0.198786 \\
\hline & & 2 & $0 \leq c_{2}<0.195808$ & $c_{2} \geq 0.195809$ & 0.195808 \\
\hline & & 2 & $0 \leq c_{2}<0.194720$ & $c_{2} \geq 0.194721$ & 0.194720 \\
\hline
\end{tabular}

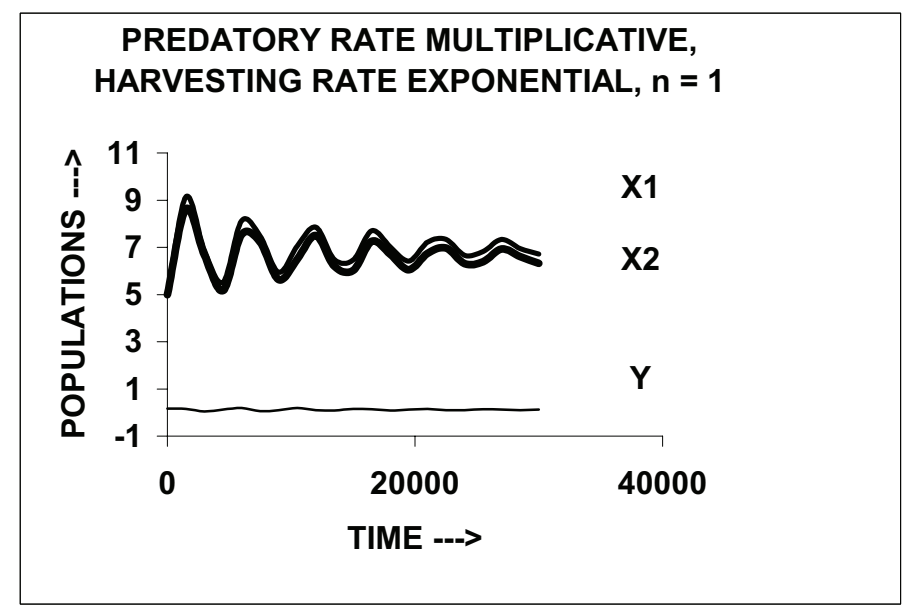

Figure 1. Population with predatory rate multiplicative, harvesting rate exponential 


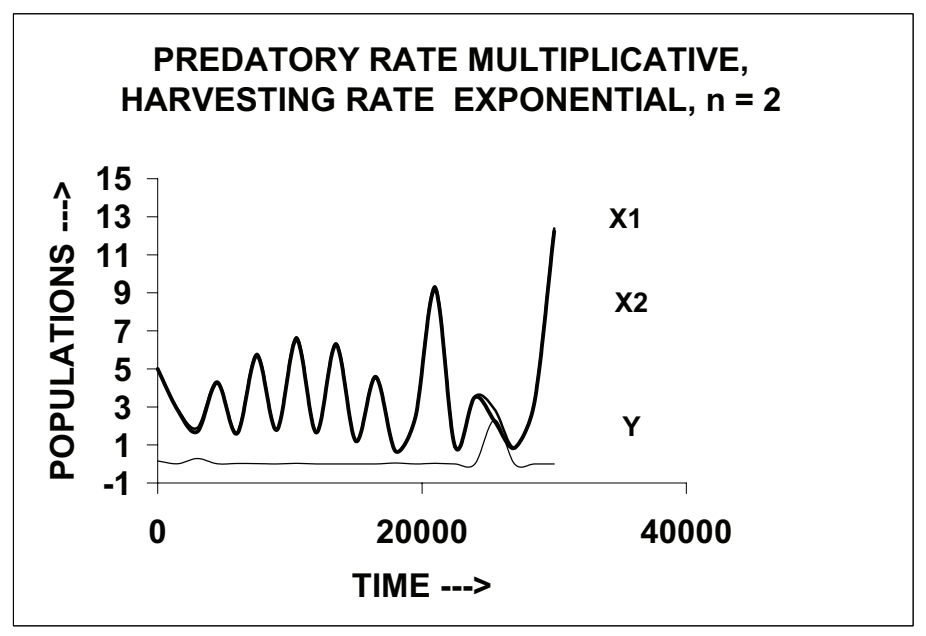

Figure 2. Population with predatory rate multiplicative, harvesting rate exponential

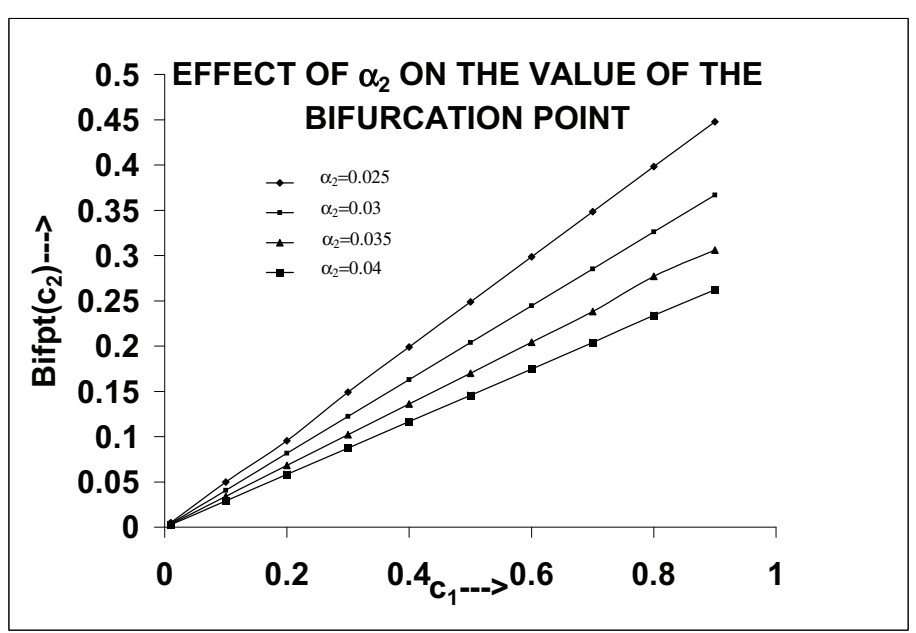

Figure 3. Population with predatory rate multiplicative, harvesting rate exponential

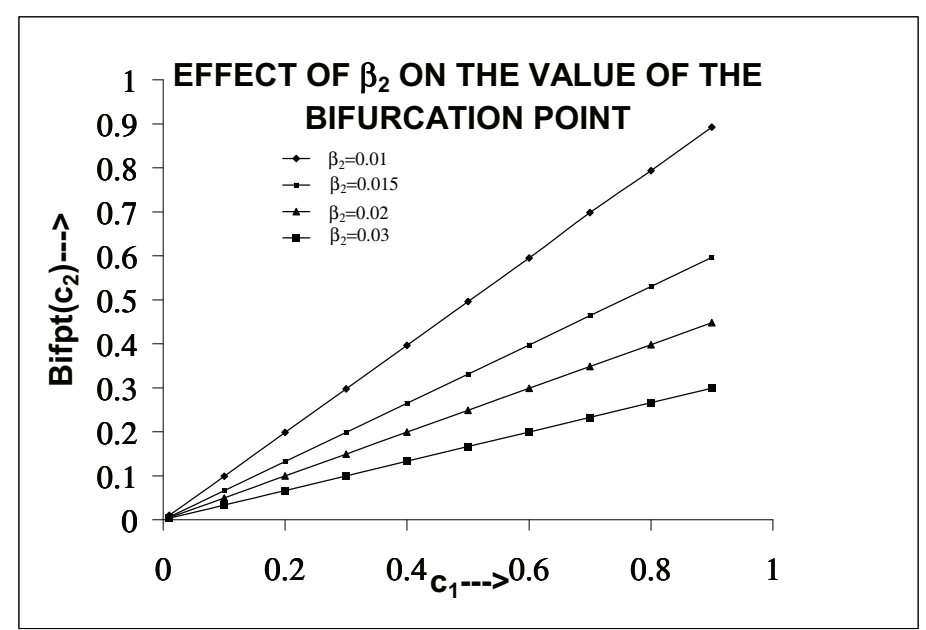

Figure 4. Population with predatory rate multiplicative, harvesting rate exponential 